\title{
A nanopore interface for high bandwidth DNA computing
}

\author{
Karen Zhang ${ }^{1} \uparrow$, Yuan-Jyue Chen ${ }^{2} \uparrow$, Kathryn Doroschak ${ }^{1}$, Karin Strauss ${ }^{2}$, Luis Ceze ${ }^{1}$, Georg \\ Seelig ${ }^{1,3,4}$, and Jeff Nivala ${ }^{1,4}$
}

${ }^{1}$ Paul G. Allen School of Computer Science and Engineering, University of Washington, Seattle, WA, USA.

${ }^{2}$ Microsoft Research, Redmond, WA, USA.

${ }^{3}$ Department of Electrical and Computer Engineering, University of Washington, Seattle, WA, USA.

${ }^{4}$ Molecular Engineering and Sciences Institute, University of Washington, Seattle, WA, USA. †these authors contributed equally.

*correspondence to: jmdn@cs.washington.edu

\begin{abstract}
DNA has emerged as a powerful substrate for programming information processing machines at the nanoscale. Among the DNA computing primitives used today, DNA strand displacement (DSD) is arguably the most popular, with DSD-based circuit applications ranging from disease diagnostics to molecular artificial neural networks. The outputs of DSD circuits are generally read using fluorescence spectroscopy. However, due to the spectral overlap of typical small-molecule fluorescent reporters, the number of unique outputs that can be detected in parallel is limited, requiring complex optical setups or spatial isolation of reactions to make output bandwidths scalable. Here, we present a multiplexable sequencing-free readout method that enables real-time, kinetic measurement of DSD circuit activity through highly parallel, direct detection of barcoded output strands using nanopore sensor array technology (Oxford Nanopore Technologies' MinION device). We show that engineered reporter probes can be detected and classified with high accuracy at the single-molecule level directly from raw nanopore signals using deep learning. We then demonstrate this method's utility in multiplexed detection of clinically relevant microRNA sequences. These results increase DSD output bandwidth by an order of magnitude over what is possible with fluorescence spectroscopy, laying the foundations for a new paradigm in DNA circuit readout and programmable multiplexed molecular diagnostics using portable nanopore devices.
\end{abstract}

\section{Main Text}

The predictability of Watson-Crick base pairing has enabled the construction of a wide range of DNA-based computing systems including amplifiers ${ }^{1}$, Boolean logic gates ${ }^{2-4}$, chemical reaction networks ${ }^{5,6}$, oscillators ${ }^{7}$, molecular diagnostics ${ }^{8}$, and neural networks ${ }^{4,9}$. These circuits rely on a basic molecular primitive called toehold-mediated DNA strand displacement (DSD) ${ }^{10,11}$. DSD is a competitive hybridization reaction in which a single-stranded DNA (ssDNA) or RNA input displaces an incumbent output strand from a complementary binding partner. Multiple DSD reactions can cascade to create a complex, yet programmable reaction network. Due to the simplicity of this mechanism and its ability to operate in both cellular and enzyme-free settings, DSD circuits are widely applied, readily scaled up, and constitute some of the largest molecular circuits designed by humanity so far. 
Readout of DNA strand displacement activity typically relies on a fluorophore-quencher strategy, in which a DSD reaction is designed to liberate a fluorophore-labeled strand from a quencher-labeled strand. The fluorescent properties of the reaction can then be measured with a spectrofluorometer to determine the relative concentration of the free fluorophore-labeled strand ${ }^{12}$. Several unique fluorophores can be combined in a single system to label different DSD components, however spectral overlap amongst fluorescent dyes (e.g. FAM, TAMRA, Cy5) ultimately limit this multiplexing to 3 or 4 unique labels. Even then, achieving this scale of orthogonal readouts requires complex and expensive instrumentation, such as multiple sets of optical filters ${ }^{13-15}$. As the field of DNA computing progresses, more scalable detection methods are critical for circuit multiplexing, multilayer kinetic characterization, and debugging ${ }^{2}$. Ideally, such readout technologies would also be inexpensive, portable, and fast, which would support applications such as rapid diagnostics.

Nanopore sensing is a simple single-molecule detection method applicable to a wide range of analytes from small molecules and peptides, to nucleic acids and proteins ${ }^{16-20}$. Nanopore array technology has recently been commercialized for DNA and RNA sequencing, in which the technology's key advantages compared to other sequencing technologies include instrument portability and real-time data streaming. Nanopore sequencing is facilitated by enzyme-assisted translocation of nucleic acid strands through the pore, making it possible to achieve singlenucleotide sequence resolution of long DNA fragments ${ }^{21}$. In this sequencing approach, a ligation step is performed prior to sequencing to attach the target strands to adapter DNA fragments bound to the requisite motor proteins. This procedure is currently incompatible for DSD circuit readout, especially when analysis of circuit kinetics is desired. Furthermore, strands in DSD circuits are usually too short (around 15-50 nt) to be reliably characterized via conventional nanopore sequencing.

Previous studies have explored non-sequencing-based nanopore sensing techniques for nucleic acid strand detection in the context of DNA computing ${ }^{22}$. For example, nanopore detection of unlabeled DNA and RNA circuit outputs has been demonstrated with micro-droplet systems, wherein the target strand is electrophoretically pulled through a protein pore connecting two droplets $^{23-25}$. However, such studies have not yet shown these systems to be quantifiable nor multiplexable. Meanwhile, solid-state nanopores have been used to characterize the doublestranded outputs of DNA assembly reactions, but it is unclear whether they would be suitable for the short single-stranded outputs of typical DSD circuits ${ }^{26}$. Apart from DNA computing, nanopore technology has also been used for miRNA detection, facilitating disease diagnostics ${ }^{22,27}$. Further studies in this area demonstrated that peptide nucleic acid (PNA) and polyethylene glycol (PEG) probes are effective at targeting specific miRNAs for nanopore detection ${ }^{28,29}$. However, it remains challenging to multiplex detection of multiple miRNAs using these probes.

Here, we developed a multiplexable reporting strategy that utilizes off-the-shelf nanopore sensing array technology (Oxford Nanopore's MinION) ${ }^{21}$ to dynamically monitor DSD circuit activity in real-time, enabling more scalable readout of output kinetics using an inexpensive, portable device. First, we demonstrate successful kinetic characterization of a single DSD circuit operating within a nanopore array flow cell with results comparable to traditional fluorescencebased reporting systems. We then design and characterize dozens of nanopore barcodes that can be used for multiplexed DSD circuit reporting. In this strategy, barcodes are classified directly from raw nanopore signal data using machine learning. Finally, we implement this reporting strategy to discriminate amongst combinations of three single nucleotide variants (SNVs) of the let-7 microRNA family. 
To develop our reporting method, we first designed a modified DNA strand architecture that would allow us to sense ssDNA DSD outputs using a nanopore (Fig. 1a). We did this by modifying the output strand to include a 3' biotin modification. We reasoned that, when conjugated to streptavidin, this modification would prevent displaced ssDNA from fully translocating through the nanopore after its free 5' end is electrophoretically captured. The nanopore ionic current signal would then be dependent on the sequence of the strand immobilized within the pore ${ }^{17}$. To verify this approach, we tested biotin-modified DNA strands in the presence of streptavidin on a MinION nanopore sensor array using a custom run script that briefly reversed the applied voltage across the nanopore array every ten seconds, allowing us to repeatedly capture strands from the bulk solution and then eject them electrophoretically ${ }^{30}$. Repeatedly sampling strands from the bulk flow cell solution in this manner allowed us to detect DNA-specific nanopore capture events at a concentration-dependent frequency (Fig. 1b-d).

After confirming that we could detect free biotin-streptavidin-modified ssDNA strands, we next tested whether this technique could be coupled to sense the free output strand concentration of a catalytic seesaw-based DSD reaction (Fig. 2a and b). Seesaw circuits have previously been used to build large-scale logic circuits and neural networks ${ }^{2,4,9}$. In this circuit architecture (Circuit 1 with Barcode A1, see Supplementary Tables S1 and S2), an input strand displaces a gate-bound output strand. A fuel strand then binds to the gate and displaces the input, freeing it to trigger more of the output. As such, seesaw gates can catalytically amplify its input, a critical step for restoring signals. To allow detection of the seesaw gate output strand, we designed the strand with the requisite 3 ' biotin modification, in addition to a short polyT linker between this modification and the rest of the sequence. We then hybridized the output strand with the complementary gate strand to form the gate complex. The gate complex bound with streptavidin was then added to a MinION flow cell in two different conditions: with and without the presence of an input strand. Results from these experiments are shown in Figure 2c. Specifically, we determined the output strand's average nanopore strand capture frequency in 5-minute intervals, allowing us to measure the reaction kinetics directly within the flow cell solution (Methods). In parallel, we also monitored an identical reaction using a traditional fluorophore-quencher-based reporter gate and fluorometer (Fig. $2 \mathrm{~b}$ and c). We found that nanopore samples had comparable output kinetics to those measured using the fluorophore-quencher approach, indicating that our nanopore method can be used to accurately monitor the reaction kinetics of a catalytic DSD reaction. We also noted that samples with no input added showed higher levels of output strand "leak" when using the fluorescent reporting method. We hypothesized that this circuit leakage was caused by the interaction between the seesaw gate and the fluorescent reporter gate ${ }^{2}$ (Fig. 2b). Because our nanopore readout strategy detects the ssDNA output strand directly, it does not require an additional reporter gate, thus the reporter leak was not observed on the nanopore-based kinetics plot. To verify this hypothesis, we tested a "clamped" seesaw gate (Supplementary Table S3) that has previously been shown to suppress reporter leakage ${ }^{2}$. Indeed, the no input sample kinetics using a clamped seesaw gate more closely matched the no input nanopore readout (Supplementary Fig. S1).

Having developed a technique to measure DSD kinetics of a single reaction using a nanopore array, we next sought to explore multiplexed detection through barcoding of the output strand. We started by determining the nanopore-addressable region of the output strand. To do this, we designed and tested a series of ssDNA output strands that contained a single nucleotide variation at a different position within a nine-nucleotide window directly upstream of the biotinylated 3' end. We found that mutations within a six-nucleotide subset of that window manifested substantial changes to the mean ionic current signal relative to the original output 
strand (Fig. 3a and Supplementary Fig. S2). We designated this sensitive sequence region as the output strand's nanopore-addressable barcode.

As an initial test to see how accurately barcodes of dissimilar sequences could be discriminated amongst each other, we randomly addressed a set of ten different output strands (Circuits 0-9 with Barcodes A1-A9 respectively, see Supplementary Tables S1 and S2) and characterized them on a MinION. Capture event fractional mean currents for each of these barcodes showed that while some of the barcodes displayed distinct signal levels, indicating high separability based on this feature alone, some barcodes had significant overlap (Fig. $3 \mathrm{~b}$ and Supplementary Fig. S3). Fitting a logistic regression model to these means yielded a singlemolecule classification accuracy of $\sim 32 \%$ on a withheld test set (Methods). To capture more signal information that could allow for better discrimination amongst the different barcodes, we used a Convolutional Neural Network (CNN) to conduct learned feature extraction and classification directly from the raw nanopore signal (Fig. 3c and Methods). CNN models trained on data collected for each of the random barcodes yielded a single-molecule classification accuracy of $\sim 72 \%$ on a withheld test set (Fig. $3 \mathrm{~d}$ and Methods). We then chose two barcodes from this set (barcodes A0 and A5) and tested them as output reporters for two orthogonal seesaw gates (Fig. 3e). We did this by mixing the two seesaw circuits (Circuits 0 and 5) in solution and then selectively triggering one or the other with the addition of their corresponding input strand, depending on the experimental condition, immediately prior to loading into a MinION flow cell. We then quantified each circuit's reaction kinetics over the course of the experiment using the CNN to demultiplex the output strand capture signals. Results from these experiments showed that only the selectively triggered circuit barcode in each of the two experimental conditions had an elevated output signal, indicating that our classifier was able to correctly identify the barcoded circuits that were present and activated against a background of barcodes that were unactivated or not present in the sample (Figure 3e).

To further develop the multiplexing potential of this method, we next tested two sets of rationally-designed barcodes (Sets B and C, see Supplementary Table 2). Set B was based on a predictive nanopore signal model of DNA kmers ${ }^{31}$ which we used to select a set of thirteen 6-kmer barcode sequences predicted to have nearly non-overlapping ionic current levels (Supplementary Fig. S4). Set $C$ included the use of abasic sites within the barcode sequence. We reasoned that abasic sites, which lack a nitrogenous base, would yield higher ionic current levels than barcodes composed entirely of standard bases, stretching the dynamic range of barcode signal space. In total, we characterized 36 different barcodes which we were able to classify amongst each other with a non-trivial single molecule accuracy of $\sim 60 \%$ using a CNN (Fig. $4 \mathrm{a}$ and Methods). From this collection, we identified a subset of ten barcodes with the most separable signal levels (Fig. $4 \mathrm{~b}$ and Supplementary Fig. S5), and achieved an average single-molecule classification accuracy of $\sim 82 \%$ after training and testing a CNN on this limited set (Fig. 4c). We then performed another set of multiplexed seesaw circuit experiments using this subset of optimized barcodes and the corresponding CNN classifier. In these experiments, up to three circuits were activated at a time in samples containing a total of five orthogonal seesaw circuits. In contrast to our previous nanopore experiments, input strands were introduced into the samples and the reactions were allowed to reach steady state prior to analysis on the nanopore (Methods). Specifically, our classifier was tasked with distinguishing amongst all ten barcodes in our optimized set, although only five of those (barcodes B10, B7, C13, C8, and C12) were physically present in the samples, and either two (barcodes B7 and C12) or three (barcodes B10, C13, and C8) of these circuits were activated with their corresponding input strands, depending on the experimental conditions. 
Results from these experiments are shown in Figure 4d. We found that circuits with barcodes that were activated in each sample showed elevated capture levels compared to all other barcodes. These results demonstrate the ability of our nanopore reporting strategy to multiplex DSD circuit readouts beyond what is possible with current fluorescence-based technologies.

Finally, we sought to test our reporter system in a multiplexed diagnostic application by discriminating amongst combinations of single nucleotide variants (SNVs) of the let-7 microRNA (miRNA) family. The let7 family of miRNAs play an important role in human development and can be used as biomarkers for disorders such as cancer ${ }^{32}$. Inspired by Chen et al. ${ }^{33}$, we designed competitive three-stranded probes for the detection of three variants: let-7a, let-7e, and a truncated version of let-7c (Fig. 5a and b, also Supplementary Table S4). Let-7c was truncated to reduce potential probe crosstalk by destabilizing an undesired wobble base pairing between let-7c miRNA and let-7a probe. We chose three barcodes from our designed set and assigned a different one to each variants' probe. If a particular miRNA variant is present, its corresponding probe should be triggered, allowing its barcoded output strand to be detected by the nanopore sensor array. We tested this strategy with experiments containing all three probes in a single reaction solution, which we then challenged with different combinations of let-7a, let-7c, and let-7e RNA inputs prior to measurement with a MinION. Results from these experiments showed clear enrichment of barcodes corresponding to the probes that were triggered by their cognate RNA input (Fig. 5c). Although mostly orthogonal, RNA/probe crosstalk was observed in the case where addition of let7 e input triggers release of let-7a probe output slightly above background levels. This is likely due to the relatively large distance between the let-7e SNV and toehold-complementary region (T1*), which lowers the kinetic penalty of let-7e inputs hybridizing to let-7a probes. These results are also consistent with fluorescent reporter-based measurements, indicating that this crosstalk is not a result of our nanopore-based reporters. (Supplementary Fig. S6). Overall, these results demonstrate successful multiplexing of a microRNA-based diagnostic.

In summary, we have developed a new reporter strategy for DSD reactions using nanopore sensing. Our system holds key advantages over fluorescence-based methods, including greater multiplexing and real-time readout using an inexpensive, portable device with flow cells that can be re-used for multiple analytical samples (Supplementary Fig. S7). Future work will be aimed at: 1) further expansion of the barcode space, for example, with chemical modifications to the DNA that could expand the dynamic range of barcode signal space, and/or the ability to read sequential barcode regions within a single output strand ${ }^{24,34,35}$; and 2) increasing the reaction speed and sensitivity of DSD reactions, for example by spatially-localizing DNA components to the nanopore sensor membrane ${ }^{36,37}$. Increasing the scale and speed of our detection strategy for more complex molecular computing architectures, such as cascaded circuits or oscillators, will take further advantage of our method's ability to generate both multiplexed and kinetic readouts. 
Acknowledgements: We thank members of the Molecular Information Systems Lab for helpful discussion and feedback on this work. We also thank Oxford Nanopore Technologies for providing the configurable MinION run script and discussions on its use.

Funding: This work was supported in part by NSF EAGER Award 1841188 and NSF CCF Award 2006864 to LC and JN, NSF CCF Award 1954665 to GS and JN, and a sponsored research agreement from Oxford Nanopore Technologies.

Author contributions: K.Z. performed wet lab experiments. K.Z. and K.D. developed the data analysis pipeline and performed computational analyses. K.Z., Y.C., G.S., and J.N. designed experiments. Y.C., K.S., L.C., G.S., and J.N. supervised the project. J.N. conceived and directed the project. All authors contributed to writing and editing of the manuscript.

Competing interests: Y.C. and K.S. are employees of Microsoft. J.N. is a consultant to Oxford Nanopore Technologies. The remaining authors declare no competing interests. 


\section{Methods}

\section{$\underline{\text { MinION experiments }}$}

All MinION runs were performed using R9.4.1 flow cells (Oxford Nanopore Technologies). All runs were configured at a temperature of $30{ }^{\circ} \mathrm{C}$, bias voltage of $-180 \mathrm{mV}$, sampling frequency of $10 \mathrm{kHz}$, and static flip frequency of $15 \mathrm{sec}$ with the use of a custom MinKNOW script (available from ONT) which allows for collection of raw current data. All samples were suspended in 1X C17 buffer (2 M KCl, $100 \mathrm{mM}$ HEPES, pH 8). Samples were pipetted into the flow cell priming port. If running multiple samples on the same flow cell, the flow cell was washed with $3 \mathrm{~mL} 1 \mathrm{X} \mathrm{C17}$ for $5 \mathrm{~min}$ between samples. When not in use, flow cells were stored at $4{ }^{\circ} \mathrm{C}$ in $\mathrm{C} 18$ buffer $(150 \mathrm{mM}$ potassium ferrocyanide, $150 \mathrm{mM}$ potassium ferricyanide, $25 \mathrm{mM}$ potassium phosphate, $\mathrm{pH} 8$ ).

\section{DSD circuit preparation}

All DSD circuit components were ordered from Integrated DNA Technologies (IDT). All components were stored at $-20{ }^{\circ} \mathrm{C}$ in the long term and $4{ }^{\circ} \mathrm{C}$ in the short term (less than a month). Circuit gate complexes were constructed by mixing 4 nmol output strand with 4 nmol bottom strand in $0.8 \mathrm{X} \mathrm{C17}$. Strands were annealed in a thermocycler starting at $95{ }^{\circ} \mathrm{C}$ for 2 min and decreasing $1{ }^{\circ} \mathrm{C}$ every 1 min cycle for 75 cycles. New England Biolabs (NEB) Purple Gel Loading Dye (no SDS) was added to annealed product at a final concentration of $1 \mathrm{X}$. The double-stranded annealed product was then separated from excess ssDNA on a $10 \%$ non-denaturing polyacrylamide gel. Bands corresponding to annealed product were cut and submerged in $1 \mathrm{X} \mathrm{C17}$ for at least 24 hours to elute.

Fluorescent reporters were prepared by mixing quencher strands at $30 \%$ higher concentration than fluorophore strands in $1 \mathrm{X} \mathrm{C17}$. Strands were annealed using the thermocycler protocol described above.

\section{Titration experiments}

The standard curve is an average of titration data from three different output strands. In each titration experiment, the output strand was run on the MinION at concentrations of $0.02,0.1$, $0.2,0.5$, and $1 \mathrm{uM}$. Each sample also contained streptavidin (NEB \#N7021S) at $4 \mathrm{uM}$ and was suspended in $1 \mathrm{X} \mathrm{C} 17$. To ensure enough captures were collected for data analysis at low concentrations, the $0.02 \mathrm{uM}$ sample was run for $20 \mathrm{~min}$, the $0.1 \mathrm{uM}$ sample for $15 \mathrm{~min}$, and the rest of the samples for $10 \mathrm{~min}$.

\section{$\underline{\text { Kinetics analysis of a single DSD circuit }}$}

The sample for this experiment consisted of $0.5 \mathrm{uM}$ gate complex, $2 \mathrm{uM}$ streptavidin, and $2 \mathrm{uM}$ fuel strand suspended in $1 \mathrm{X} \mathrm{C17}$ to a total volume of $200 \mathrm{uL}$. Immediately prior to loading into the MinION flow cell, $0.2 \mathrm{uM}$ input strand was added to initiate the reaction. Ionic current data was collected over the course of four hours on the MinION. The average time between captures was calculated for each five minute interval throughout the run and converted into a predicted concentration via our standard curve.

Fluorescence kinetics data of the same circuit at a total volume of $100 \mathrm{uL}$ was collected every $1 \mathrm{~min}$ using a microplate reader (Synergy HTX, Multi-Mode reader, Biotek). Excitation (emission) wavelengths were $485 \mathrm{~nm}(528 \mathrm{~nm})$ for dye FAM and $620 \mathrm{~nm}(680 \mathrm{~nm})$ for dye Cy5. 
Sample composition was identical to the nanopore sample except for the addition of a fluorescent reporter gate at $3 \mathrm{uM}$.

\section{Multiplexing experiments}

Samples for the two-circuit multiplexing experiment consisted of $0.5 \mathrm{uM}$ gate complex and $2 \mathrm{uM}$ fuel strand from each circuit, along with a total of $4 \mathrm{uM}$ streptavidin, suspended in 1X C17 to a total volume of $200 \mathrm{uL}$. Immediately prior to loading into the MinION flow cell, $0.2 \mathrm{uM}$ input for each circuit was added.

Samples for the five-circuit multiplexing experiment consisted of $0.2 \mathrm{uM}$ gate complex from each circuit and a total of $3.2 \mathrm{uM}$ streptavidin suspended in 1X C17 to a total volume of 200 uL. $0.4 \mathrm{uM}$ of each desired input was added to the samples, which were then immediately placed in a $30{ }^{\circ} \mathrm{C}$ incubator and allowed to react to steady state over the course of three hours. Samples were then loaded into a MinION flow cell for analysis. Each sample was run for $10 \mathrm{~min}$.

\section{Let-7 probe preparation}

All let-7 probe components were ordered from IDT. Helper strands and fuel strands were PAGE purified while all other strands were HPLC purified. All components were stored at $-20{ }^{\circ} \mathrm{C}$ in the long term and $4{ }^{\circ} \mathrm{C}$ in the short term (less than a month). Probes were constructed by mixing top and output strands each at $20 \%$ higher concentration than bottom strands in $1 \mathrm{X} \mathrm{C17}$. Strands were annealed in a thermocycler starting at $95{ }^{\circ} \mathrm{C}$ and decreasing $1{ }^{\circ} \mathrm{C}$ every 1 min cycle for 75 cycles. Glycerol was added to annealed products at a final concentration of $10 \%$ by volume. The annealed complex was then separated from excess ssDNA on a $10 \%$ non-denaturing polyacrylamide gel. Bands corresponding to annealed product were cut and submerged in $1 \mathrm{X} \mathrm{C17}$ for at least 24 hours to elute.

\section{Let-7 detection experiments}

Samples for multiplexed detection of let-7 SNVs consisted of $100 \mathrm{nM}$ of each let-7 probe, $130 \mathrm{nM}$ of each let-7 helper strand, and $1200 \mathrm{nM}$ streptavidin suspended in 1X C17 to a total volume of $200 \mathrm{uL} .50 \mathrm{nM}$ of each desired let-7 microRNA was added to the samples, which were then immediately placed in a $30{ }^{\circ} \mathrm{C}$ incubator and allowed to react to steady state over the course of one hour. Samples were then loaded into a MinION flow cell for analysis. Each sample was run for $10 \mathrm{~min}$.

Fluorescence kinetics data of the same circuit at a total volume of $100 \mathrm{uL}$ was collected every $1 \mathrm{~min}$ using a microplate reader (Synergy HTX, Multi-Mode reader, Biotek). Excitation (emission) wavelengths were $485 \mathrm{~nm}(528 \mathrm{~nm})$ for dye FAM and $620 \mathrm{~nm}(680 \mathrm{~nm})$ for dye Cy5.

Nanopore data analysis

The nanopore data analysis pipeline is adapted from Cardozo et al. ${ }^{30}$ and begins by isolating capture events from raw nanopore current. A capture event occurs when the nanopore current drops to $70 \%$ or below of its open pore level for longer than one millisecond. The fractional current throughout each capture event is then calculated, which is defined as the current observed during the capture event $\left(I_{b}\right)$ divided by the open pore current $\left(I_{o}\right)$. To separate putative output strand capture events from background noise, each capture event is passed through a filter that checks whether five of its signal features (mean, median, minimum, maximum, standard deviation) are within the expected range. Capture events are also passed through a length filter which discards events that have a capture duration of less than two seconds. 
Initial exploration of classification on Barcode Set A was performed using the LogisticRegression model from scikit-learn. The model was fitted to at least 14000 examples per class using a 80/20 train/test split. Classification accuracy is defined as the number of correct predictions (true positives + true negatives) divided by total predictions, converted to a percentage.

All future classification of capture events is performed by a Convolutional Neural Network (CNN) via PyTorch. For each capture event, the first two-second window of its raw nanopore signal is reshaped into a 2D array before being fed into the CNN. The CNN comprises four convolutional layers each with ReLU activation and max pooling, followed by a fully connected layer with log-sigmoid activation and an output layer containing a neuron for each barcode class. Reported accuracies and confusion matrices come from test results of the CNN after training with at least 3000 examples per class using a 80/20 train/test split for 250 epochs.

Capture events from a particular class are quantified using a standard curve (determined from titration experiments) that relates the average time between capture events to sample concentration. The time between two capture events at a given pore is calculated by subtracting the end time of the first capture from the start time of the subsequent capture. In addition, any time periods between these capture events where the pore was occupied with noise or other barcode capture events (as determined by our filter and classifier) are also subtracted. The average time between captures is calculated from an aggregate list of total time between captures from all functional pores in a given experiment. Capture events can also be quantified using capture frequency, described in the next section.

\section{Capture frequency quantification}

Capture frequency $f$ is defined as the number of reads for a given barcoded strand per good channel per minute. It is normalized using the following equation, where $f_{\text {background }}$ and $f_{\text {saturated }}$ represent the capture frequency of the barcoded strand when its circuit is unactivated (no input) and when it is activated to steady state respectively (these values were determined in separate experiments).

$$
f_{\text {normalized }}=\frac{f-f_{\text {background }}}{f_{\text {saturated }}-f_{\text {background }}}
$$

\section{Code Availability}

The data analysis pipeline code used for extracting, filtering, classifying, and quantifying nanopore capture events can be found at https://github.com/uwmisl/dna-nanopore-computing. Custom MinION MinKNOW run scripts can be obtained from Oxford Nanopore Technologies upon request. 


\section{References}

1. Zhang, D. Y., Turberfield, A. J., Yurke, B. \& Winfree, E. Engineering entropy-driven reactions and networks catalyzed by DNA. Science. 318, 1121-1125 (2007).

2. Qian, L. \& Winfree, E. Scaling up digital circuit computation with DNA strand displacement cascades. Science. 332, 1196-1201 (2011).

3. Seelig, G., Soloveichik, D., Zhang, D. Y. \& Winfree, E. Enzyme-free nucleic acid logic circuits. Science. 314, 1585-1588 (2006).

4. Cherry, K. M. \& Qian, L. Scaling up molecular pattern recognition with DNA-based winner-take-all neural networks. Nature 559, 370-388 (2018).

5. Soloveichik, D., Seelig, G. \& Winfree, E. DNA as a universal substrate for chemical kinetics. Proc. Natl. Acad. Sci. U. S. A. 107, 5393-5398 (2010).

6. Chen, Y. J. et al. Programmable chemical controllers made from DNA. Nat. Nanotechnol. 8, 755-762 (2013).

7. Srinivas, N., Parkin, J., Seelig, G., Winfree, E. \& Soloveichik, D. Enzyme-free nucleic acid dynamical systems. Science (80-. ). 358, (2017).

8. Zhang, C. et al. Cancer diagnosis with DNA molecular computation. Nat. Nanotechnol. 2020158 15, 709-715 (2020).

9. Qian, L., Winfree, E. \& Bruck, J. Neural network computation with DNA strand displacement cascades. Nature 475, 368-372 (2011).

10. Zhang, D. Y. \& Winfree, E. Control of DNA strand displacement kinetics using toehold exchange. J. Am. Chem. Soc. 131, 17303-17314 (2009).

11. Zhang, D. Y. \& Seelig, G. Dynamic DNA nanotechnology using strand-displacement reactions. Nat. Chem. 3, 103-113 (2011).

12. Yurke, B., Turberfield, A. J., Mills, A. P., Simmel, F. C. \& Neumann, J. L. A DNAfuelled molecular machine made of DNA. Nat. 20004066796 406, 605-608 (2000).

13. Y, W. et al. Rapid Sequential in Situ Multiplexing with DNA Exchange Imaging in Neuronal Cells and Tissues. Nano Lett. 17, 6131-6139 (2017).

14. Guo, J., Wang, S., Dai, N., Teo, Y. N. \& Kool, E. T. Multispectral labeling of antibodies with polyfluorophores on a DNA backbone and application in cellular imaging. Proc. Natl. Acad. Sci. 108, 3493-3498 (2011).

15. J, J., C, R., CW, F., AN, G. \& RA, M. Fluorescence energy transfer dye-labeled primers for DNA sequencing and analysis. Proc. Natl. Acad. Sci. U. S. A. 92, 43474351 (1995).

16. Ashkenasy, N., Sánchez-Quesada, J., Bayley, H. \& Ghadiri, M. R. Recognizing a single base in an individual DNA strand: A step toward DNA sequencing in nanopores. Angew. Chemie - Int. Ed. 44, 1401-1404 (2005).

17. Stoddart, D., Heron, A. J., Mikhailova, E., Maglia, G. \& Bayley, H. Single-nucleotide discrimination in immobilized DNA oligonucleotides with a biological nanopore. Proc. Natl. Acad. Sci. U. S. A. 106, $7702-7707$ (2009).

18. Gu, L. Q., Braha, O., Conlan, S., Cheley, S. \& Bayley, H. Stochastic sensing of organic analytes by a pore-forming protein containing a molecular adapter. Nature 398, 686-690 (1999).

19. Rotem, D., Jayasinghe, L., Salichou, M. \& Bayley, H. Protein detection by nanopores equipped with aptamers. J. Am. Chem. Soc. 134, 2781-2787 (2012).

20. Ouldali, H. et al. Electrical recognition of the twenty proteinogenic amino acids using an aerolysin nanopore. Nature Biotechnology vol. 38 176-181 (2020). 
21. Jain, M., Olsen, H. E., Paten, B. \& Akeson, M. The Oxford Nanopore MinION: delivery of nanopore sequencing to the genomics community. Genome Biol. 17, 111 (2016).

22. Ding, T. et al. DNA nanotechnology assisted nanopore-based analysis. Nucleic Acids Res. 48, 2791-2806 (2020).

23. Ohara, M., Takinoue, M. \& Kawano, R. Nanopore Logic Operation with DNA to RNA Transcription in a Droplet System. ACS Synth. Biol. 6, 1427-1432 (2017).

24. Ohara, M., Sekiya, Y. \& Kawano, R. Hairpin DNA unzipping analysis using a biological nanopore array. Electrochemistry 84, 338-341 (2016).

25. Yasuga, H. et al. Logic gate operation by DNA translocation through biological nanopores. PLoS One 11, e0149667 (2016).

26. Zhu, Z., Wu, R. \& Li, B. Exploration of solid-state nanopores in characterizing reaction mixtures generated from a catalytic DNA assembly circuit. Chem. Sci. 10, 1953-1961 (2019).

27. Wang, Y., Zheng, D., Tan, Q., Wang, M. X. \& Gu, L. Q. Nanopore-based detection of circulating microRNAs in lung cancer patients. Nat. Nanotechnol. 6, 668-674 (2011).

28. Tian, K., He, Z., Wang, Y., Chen, S. J. \& Gu, L. Q. Designing a polycationic probe for simultaneous enrichment and detection of microRNAs in a nanopore. ACS Nano 7, 3962-3969 (2013).

29. Zhang, X., Wang, Y., Fricke, B. L. \& Gu, L. Q. Programming nanopore ion flow for encoded multiplex microRNA detection. ACS Nano 8, 3444-3450 (2014).

30. Cardozo, N. et al. Multiplexed direct detection of barcoded protein reporters on a nanopore array. Nat. Biotechnol. (2021) doi:10.1038/s41587-021-01002-6.

31. GitHub - nanoporetech/kmer_models: Predictive kmer models for development use. https://github.com/nanoporetech/kmer_models.

32. Roush, S. \& Slack, F. J. The let-7 family of microRNAs. Trends Cell Biol. 18, 505516 (2008).

33. Chen, S. X. \& Seelig, G. An Engineered Kinetic Amplification Mechanism for Single Nucleotide Variant Discrimination by DNA Hybridization Probes. J. Am. Chem. Soc. 138, 5076-5086 (2016).

34. J, M., H, V., V, V., Y, R. \& A, M. Nanopore unzipping of individual DNA hairpin molecules. Biophys. J. 87, 3205-3212 (2004).

35. G, C., J, P.-C., A, M., F, M. \& D, R.-L. Label-Free, Multiplexed, Single-Molecule Analysis of Protein-DNA Complexes with Nanopores. ACS Nano 11, 5815-5825 (2017).

36. Derrington, I. M. et al. Subangstrom single-molecule measurements of motor proteins using a nanopore. Nat. Biotechnol. 20153310 33, 1073-1075 (2015).

37. Adam, G. \& Delbrück, M. Reduction of Dimensionality in Biological Diffusion Processes. Struct. Chem. Mol. Biol. (1968). 


\section{Figures}
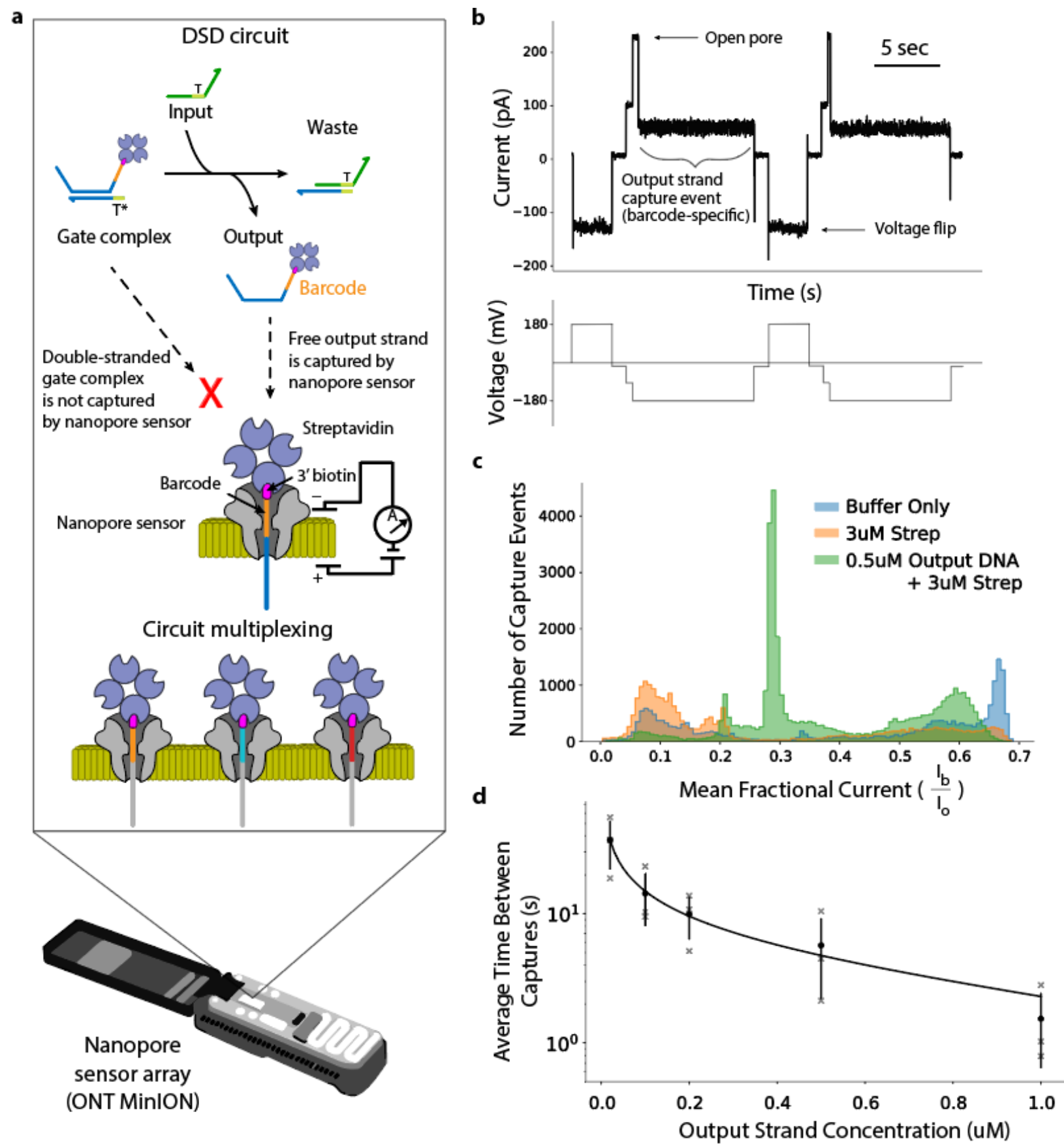

Figure 1: Nanopore detection of DNA circuit outputs. a, DSD circuit detection with a nanopore sensor. Circuit components are mixed and loaded onto a nanopore sensor array for real-time readout. Input strands react with the gate complex displacing the barcoded and 3' labeled biotinstreptavidin output ssDNA, which is then free to be captured and read by a nanopore sensor. The nanopore sensor array is capable of distinguishing different output strand barcodes, enabling circuit multiplexing. b, Example raw nanopore data showing repeated capture and ejection of biotinylated ssDNA output strands. DNA capture events manifest ionic current drops from open pore to a lower ionic current level. Strands are ejected from the pore by reversal of the applied 
voltage. c, Histogram showing the distribution of mean fractional current from capture events belonging to three samples: nanopore running buffer, running buffer with 3 uM streptavidin, running buffer with $3 \mathrm{uM}$ streptavidin and $0.5 \mathrm{uM}$ 3' biotinylated ssDNA. d, Standard curve showing the relationship between average time between output strand captures and strand concentration. Error bars show the standard deviation of three replicates, with each replicate using a different barcoded strand. 

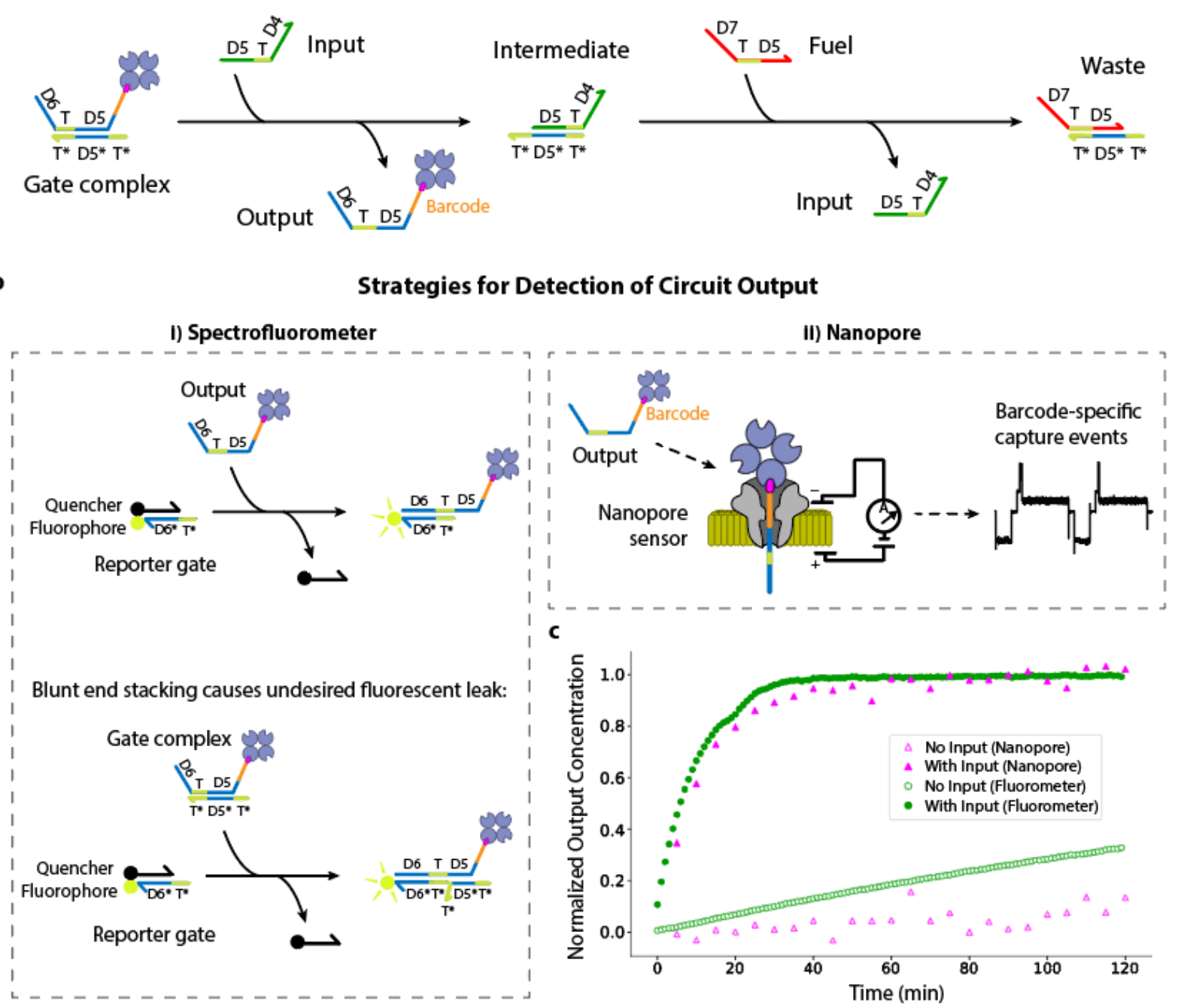

Figure 2: Nanopore and fluorometer-based comparison of catalytic DSD circuit kinetics. a, Diagram of a seesaw catalytic DSD circuit. Single-stranded input displaces the 3' labeled biotinstreptavidin single-stranded output from the gate complex, forming an intermediate with the bottom strand. The displaced output strand is now free to capture in a nanopore. Single-stranded fuel displaces input from the intermediate, recycling the input. b, Displaced output ssDNA can be read out using two detection strategies: i) Spectrofluorometer-based detection. Output strand displaces the quencher-labeled strand from a fluorophore-labeled reporter gate complex, triggering fluorescence. Blunt end stacking can occur in this reporter strategy, in which a double-stranded gate complex displaces the quencher strand, resulting in undesired "leaky" fluorescence. ii) Nanopore-based detection. Single-stranded output strand is displaced by an input strand and can then be captured in a nanopore, resulting in a detectable drop in ionic current that is diagnostic of the strand's barcode sequence. c, DSD reaction kinetics plot determined by nanopore (pink) and, for comparison, a spectrofluorometer (green), showing normalized concentration of output strand after addition of input. 
a

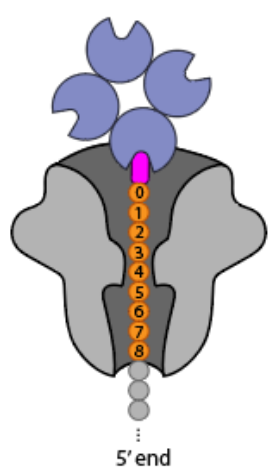

C

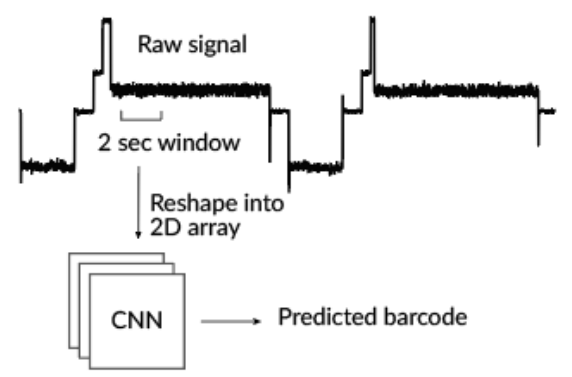

b

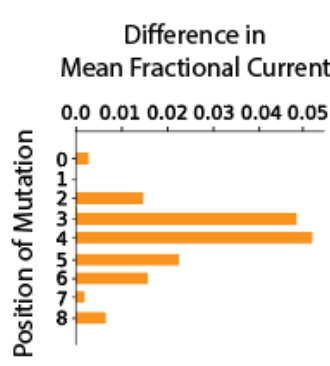

d
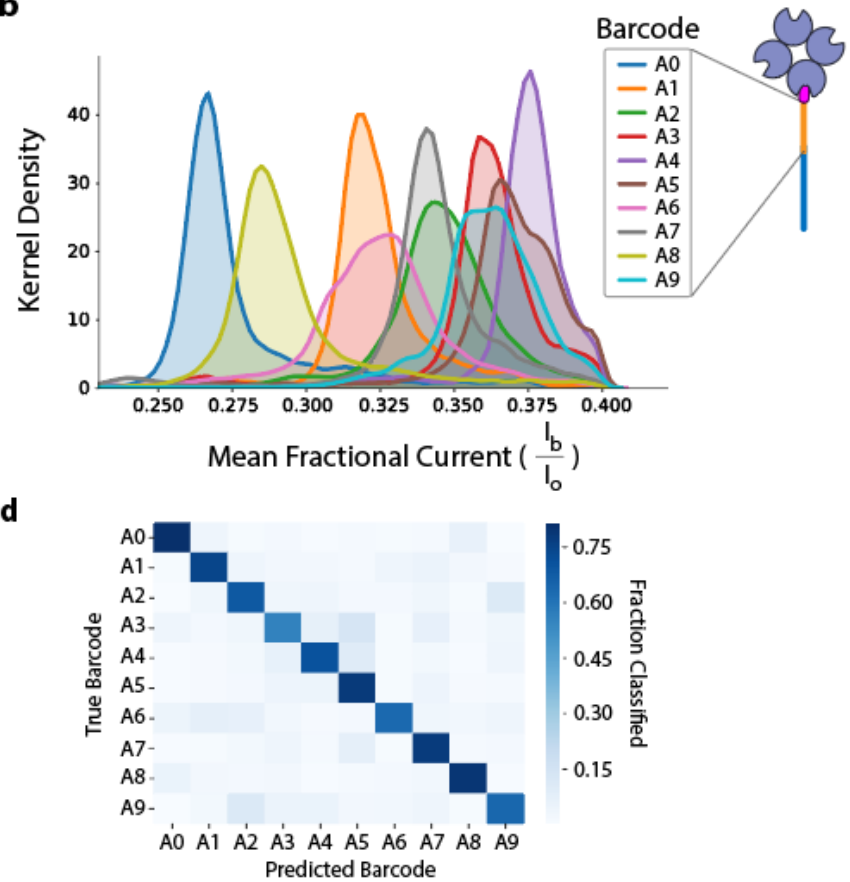

e

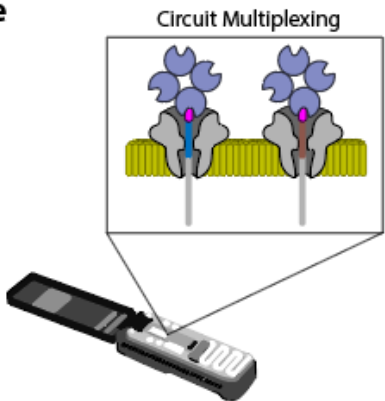

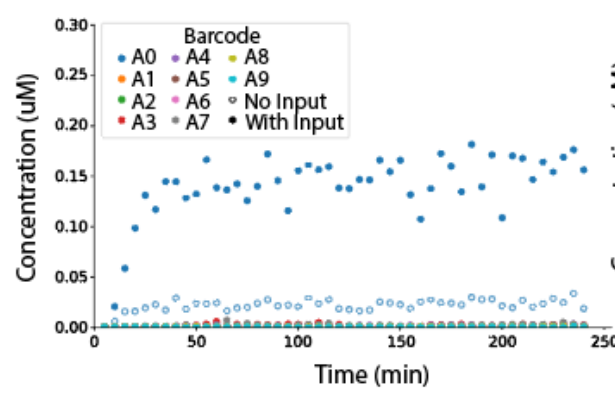

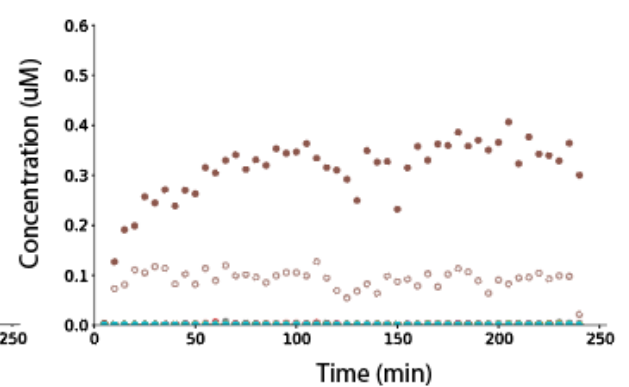

Figure 3: Initial barcode design, classification, and multiplexing. a, Mapping the output strand nanopore-sensitive region. Plot shows the absolute change in mean fractional ionic current manifested by a single-nucleotide mutation at different positions along the strand. $\mathbf{b}$, Density plot showing distribution of mean fractional current for each barcode in Set A. Each distribution is composed of $\sim 14500$ data points. c, To perform classification, a two second (20,000 data point) window of the output strand capture event signal is extracted, reshaped into a 2D array and then used as input to a 2D CNN. The CNN's output is a barcode prediction. d, Confusion matrix showing classification results of $\mathrm{CNN}$ inference on a barcode test set, which achieved an average single-molecule accuracy of $72 \%$. e, DSD reaction kinetics plot of two different circuits (barcoded with A0 or A5) multiplexed on the nanopore device. Each gate complex was present at $0.5 \mathrm{uM}$, fuel strand at $2 \mathrm{uM}$, input strand at $0.2 \mathrm{uM}$, and streptavidin at $2 \mathrm{uM}$. Two samples were prepared: No Input and With Input. Left plot shows detection of Barcode A0 output for both samples. Right plot shows detection of Barcode A5 output for both samples. 
bioRxiv preprint doi: https://doi.org/10.1101/2021.12.30.474555; this version posted December 30, 2021. The copyright holder for this preprint (which was not certified by peer review) is the author/funder, who has granted bioRxiv a license to display the preprint in perpetuity. It is made available under aCC-BY-NC-ND 4.0 International license.
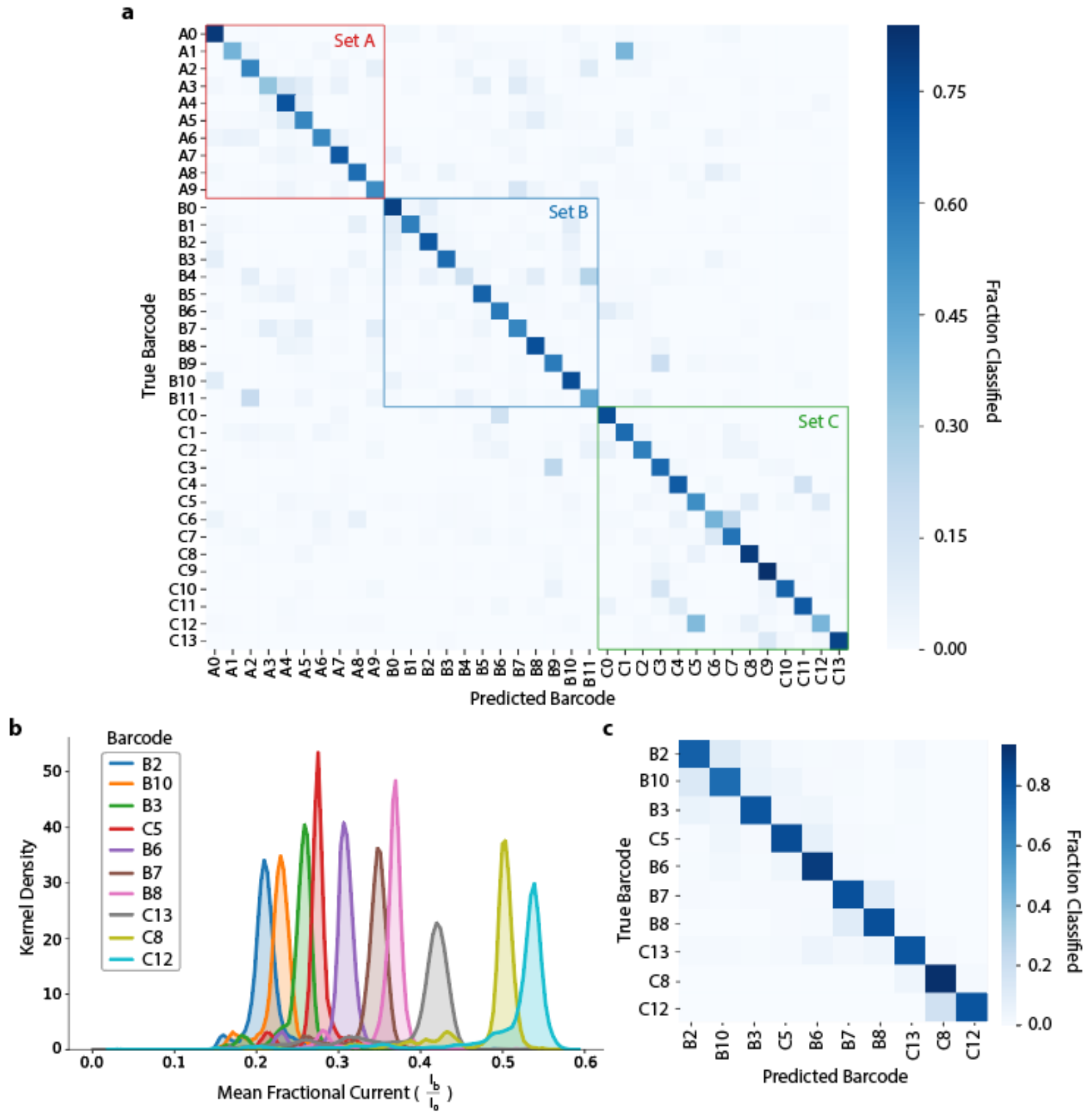

d

Activating Circuits 5 and 9

Activating Circuits 1,7 , and 8

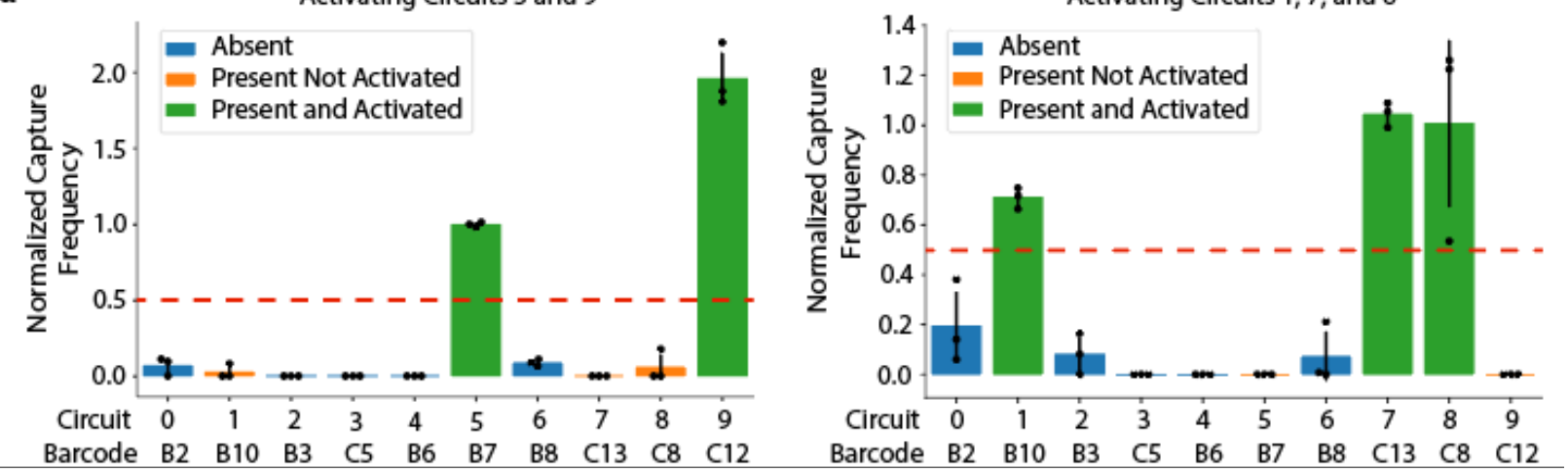


Figure 4: Developing an optimized nanopore barcode set. a, Confusion matrix showing results of CNN trained on all 36 characterized barcodes. CNN achieved an accuracy of $\sim 60 \%$ on the test set. b, Density plot showing distribution of mean fractional current for each barcode selected for the highly separable set. Each distribution is composed of $\sim 13000$ data points. c, Confusion matrix showing results of CNN trained only on the selected highly separable barcodes, which achieved an accuracy of $82 \%$ on the test set. d, Bar plots comparing normalized capture frequencies of each circuit output in two multiplexed samples. Circuits 1, 5, 7, 8, and 9 (with Barcodes B10, B7, C13, C8 and C12 respectively, see Supplementary Tables S1 and S2) were present in each sample. In the left plot, inputs for Circuits 0 and 5 were added. In the right plot, inputs for Circuits 1, 7, and 8 were added. Each gate complex was present at $0.2 \mathrm{uM}$, input at $0.2 \mathrm{uM}$, and streptavidin at 3.2 uM. Error bars show the standard deviation of three biological replicates. 


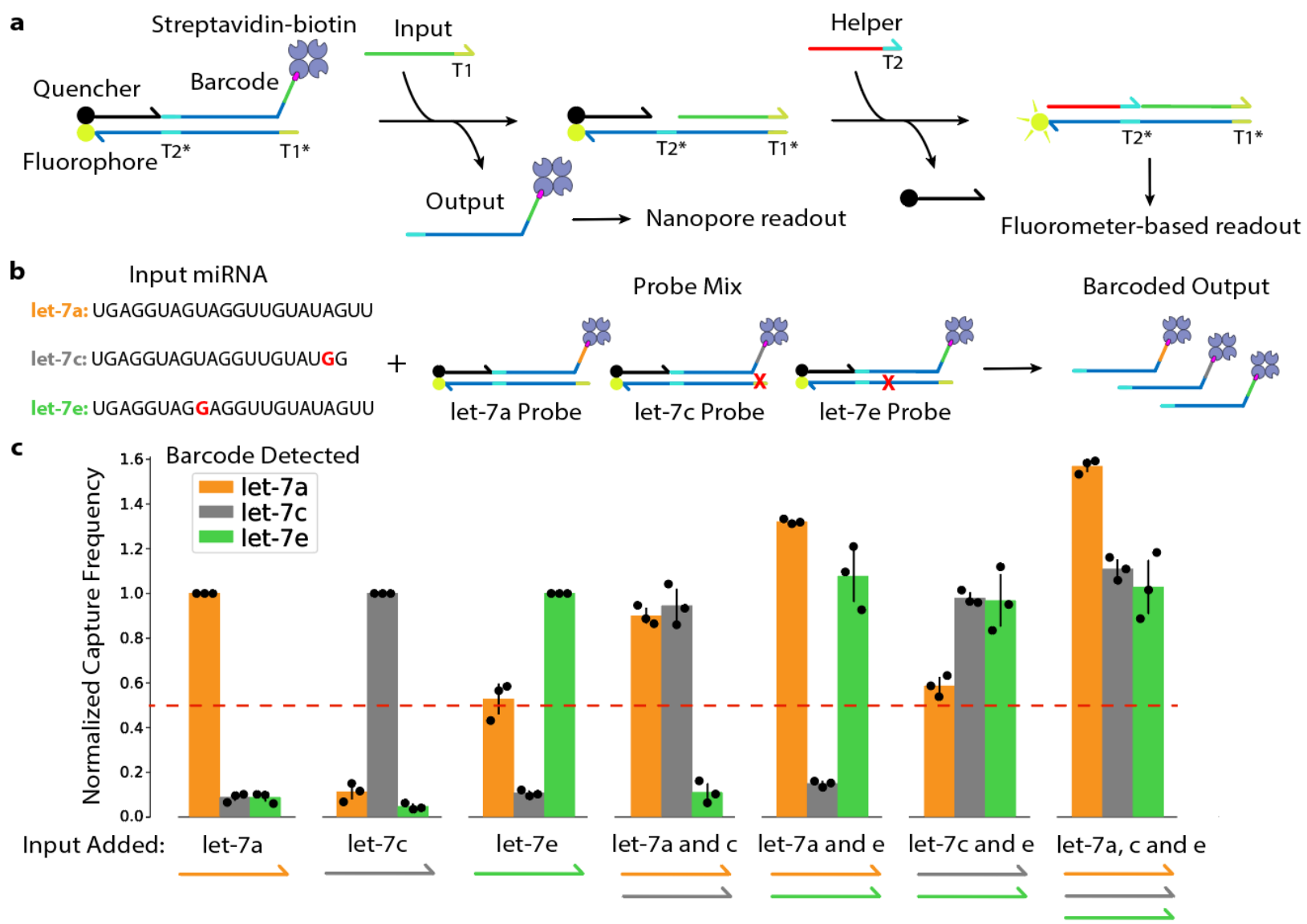

Figure 5: Multiplex detection of let-7 microRNA SNV variants. a, Two-step probe mechanism. miRNA input hybridizes to the first toehold region (T1) on the bottom strand, displacing the barcoded output strand for nanopore readout. Next, a helper strand hybridizes to the second toehold region (T2) on the bottom strand, displacing the quencher strand and enabling spectrofluorometerbased readout. b, Multiplex experiment setup. Input strands are synthetic let-7 miRNA SNV variants (sequences shown with SNVs bolded in red) which are added to a mix of probes. A red X denotes the location of bases complementary to SNVs. Hybridization of an input with the correct probe releases its respective barcoded output for nanopore detection. c, Seven samples each containing three let-7 probes were prepared. A different combination of input strands was added to each sample, as visualized under each cluster of bars. Bar plot shows the capture frequency of each barcoded output in each sample as measured on the nanopore after reaching steady state. Error bars show the standard deviation of three biological replicates. 